Via right subclavian vein, atrial and ventricular leads were advanced through and fixed in right auricle and right ventricular apex, respectively (Figure $1 C$ and $D$ ). The leads were connected to a double-chamber pacemaker.

Dextrocardia with situs inversus totalis occurs rarely, with an estimated incidence of $1: 8000-1: 10000,{ }^{1,2}$ and only $3 \%$ of the patients present with congenital heart disease. The particular technical challenge of implanting pacemaker leads in these patients is the extra angle imposed on the leads, when passing through the abnormal course of superior vena cava and reversed position of right atrium and right ventricle. Moreover, dilated right atrium resulted from atrial septal defect further increases the difficulty of implanting atrial lead. To overcome these difficulties, it may be helpful to invert the fluoroscopic image left-to-right to simulate normal anatomy, and more operation time is likely needed to successfully place leads in optimal positions.

Conflict of interest: none declared.

\title{
References
}

1. Rosenberg HN, Rosenberg IN. Simultaneous association of situs inversus, coronary heart disease and hiatus hernia. Ann Intern Med 1949;30:851-9.

2. Goyal SL, Lichestein E, Gupta PK, Chadda KD, Lajam F. Sick sinus syndrome requiring permanent pacemaker implantation in a patient with mirror-image dextrocardia. Chest 1976;69:558-61.

\section{Remote monitoring and follow-up of pacemakers and implantable cardioverter defibrillators}

\section{Haran Burri and David Senouf}

Cardiology service, University Hospital of Geneva, 23, rue Micheli-du-Crest, 1211 Geneva, Switzerland

Europace 2009;11:701-709;doi:10.1093/europace/eup110

The information given in Table 2 regarding 'Feedback to patient via transmitter' for the Boston Scientific Latitude ${ }^{\text {TM }}$ is incorrect. Feedback to the patient by the device will be given by means of coloured lights on the communicator and by indications displayed on its screen. The authors apologise for this error. 